\title{
Light fare
}

This serving of laser equipment will have to sustain readers until next year, offering a variety of lasers, software for laser measurement, a compact optical isolator for diode lasers, as well as mirror, filter and optics accessories.

Isoplot

From Uniscan

New software and image displays for the company's laser triangulation profiler

Uniscan offers analytical spatial profiling systems that include a laser triangulation-based, three-dimensional surface profiler. The three-dimensional profiler is available in the UK through AG Electro-Optics. Uniscan has announced the availability of its Isoplot Scientific graphics software for the OSP-100 profiler. According to the manufacturer, Isoplot produces high-quality, three-dimensional images of data matrices in excess of 200,000 data points. The three-dimensional views can be manipulated to show the desired features of the data and hard copy produced from the standard Windows environment. The software is Windows95 compatible and a $100 \mathrm{MHz}$ Pentium computer is required with a minimum of $16 \mathrm{MB}$ of RAM. Isoplot is capable of producing threedimensional plots where the data is shaded according to the projected light reflecting from the surface. The $z$ axis may also be amplitude colour shaded in a user-defined palette.

Reader Enquiry No. 100

\section{LBA-300PC software}

From Laser Lines

Version 1.2 of this software runs under the 32-bit mode of Windows95, which speeds beam profile access and processing

Featured in this version of the software is beam convolution, which enables the user to remove noise from both the beam view and beam calculations. Primary features of the LBA-300PC include the ability to oper-

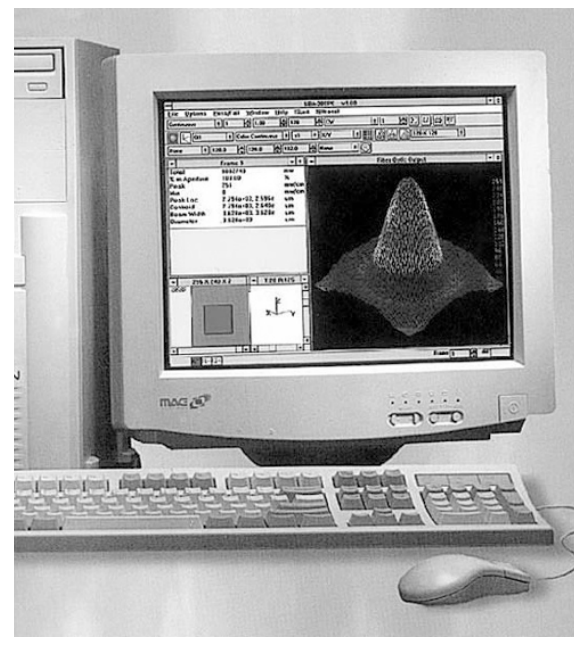

Laser Lines' LBA-300PC software works with all RS170 and CCIR cameras for laser beam testing. ate with all RS170 and CCIR cameras for laser beam diagnostics of visible and near infrared lasers. The program runs under Windows $3.1 \times$ or Windows 95 on a Pentium computer with a new high-speed PCI bus. According to the manufacturer, the most important feature of the system is an automatic calibration technique that sets the baseline of the camera precisely at zero and saves negative numbers for use in precise beam calculations. This feature allows the camera system to make secondmoment, beam-width measurements. The resolution is selectable from $512 \times 480$ down to $16 \times 15$, in steps of two. This option to change resolution allows a user to choose the needed resolution and make the trade-off between resolution and operating speed, as well as disk file space. With smaller resolution, less memory is required to store data frames. Lower resolution frames can be obtained either by zooming at single-pixel resolution to a small area, for example $512 \times 480,256 \times 240,128 \times 120,64 \times 60$, $32 \times 30$ or $16 \times 15$; or, by keeping the zoom to cover the entire sensor, reading every first, second, fourth, eighth or sixteenth pixel and line. Thus the user can obtain a low-resolution view of a large beam if it has low structure.

Reader Enquiry No. 101

\section{Dynamic Software \\ From Renishaw \\ For the measurement of critical CNC machine performance variables using both time- and distance-based capture and analysis}

Used in conjunction with the company's laser measurement system, version 3.0 of the software has a stated sampling rate of up to $5 \mathrm{kHz}$ with a resolution of $1 \mathrm{~nm}$. Data can be captured at up to $1 \mathrm{~m} \mathrm{~s}^{-1}$, which is said to be 30 per cent faster than previously available, with no additional hardware being required. Applications include condition monitoring of machines and systems involving the measurement of displacement, velocity and acceleration. The system can be used on machine tools, coordinate measuring machines, hydraulic and pneumatic systems, positioning stages, optical stages and PCB drilling machines. Using the package, it is possible to measure displacement, angle, straightness, velocity and acceleration with respect to time. The software operates under Windows (3.1, 3.11 and 95) providing support for Windows printing, cut, copy and paste functions and VGA screen support. The installer sets up the package with start-up icons in a pro-

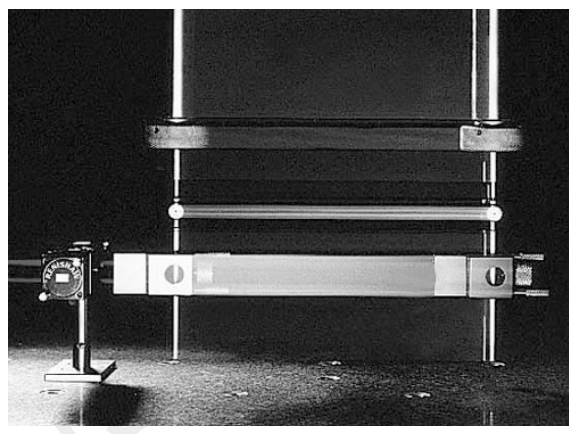

Dynamic software version 3.0 from Renishaw can be used in conjunction with the company's laser measurement system.

gram group. It is also available in Japanese, Chinese and Korean language versions.

Reader Enquiry No. 102

\section{Lasers}

Compact optical isolator for diode lasers From New Focus

Compact Faraday optical isolators designed specifically for diode lasers

These isolators are designed to provide $35-\mathrm{dB}$ peak isolation and isolate over more than $20 \mathrm{~nm}$ without requiring any adjustments. A large elliptical aperture $(3 \mathrm{~mm} \times 8 \mathrm{~mm})$ is said to eliminate the need for beam-shaping optics between the laser and the isolator. The isolator package was designed for easy mounting and compatibility with all standard mounting hardware. A half waveplate is included at the output of each isolator, giving the user complete flexibility in polarization orientation. The company's magnetic field design results in a compact $3.8 \mathrm{~cm} \times$ $3.8 \mathrm{~cm} \times 8 \mathrm{~cm}$ package and low magnetic field leakage, says New Focus.

Reader Enquiry No. 103

\section{OPC-D015-mmm=HB}

From Opto Power Corporation

A high brightness, high-power diode laser module that delivers from 13 to $15 \mathrm{~W}$ of continuous-wave (CW) power

Available at operating wavelengths ranging from $790 \mathrm{~nm}$ to $980 \mathrm{~nm}$, this compact laser is offered with a wide selection of internal focusing optics that produce working distances as long as $30 \mathrm{~mm}$ and spot sizes as small as $130 \mu \mathrm{m}$. This module is environmentally sealed and comes with an onboard monitor photodiode and a $10 \mathrm{k} \Omega$ at $25^{\circ} \mathrm{C}$ thermistor. According to the manufacturer, this laser provides an order of magnitude improvement in brightness compared to fibre-coupled diode laser arrays and 
increases the usefulness of diode lasers for numerous direct thermal and illumination applications, particularly those in industrial, medical and graphics markets that require high brightness and high power.

Reader Enquiry No. 104

\section{Verdi visible CW laser}

From Coherent

The company has released a 2-Wversion of the CW green (532 nm) diode-pumped

$\mathrm{Nd}: \mathrm{YVO}_{4}$ laser

The laser uses the same ring laser cavity as the existing $5-\mathrm{W}$ Verdi, which is stated to result in a single-frequency output with exceptionally low noise. In the $2-\mathrm{W}$ version, a single, fibre-delivered diode bar is used as the pump source, rather than the two diode bars used in the $5-\mathrm{W}$ version. Both the $2-\mathrm{W}$ and $5-\mathrm{W}$ versions are stated to be highly efficient and compact, requiring only standard 110/220 V single-phase power and no external cooling. According to the manufacturer, this solid-state technology is well suited for both scientific and industrial applications, including spectroscopy, reprographics, semiconducter inspection, holography, particle scattering, disk-zone texturing and medical applications.

Reader Enquiry No. 105

\section{GreenLine 1000}

From Edinburgh Instruments

An all-solid-state, high-power, single-

frequency laser with the diode-pumped

Nd:YLF laser

The company has broken the 1-W barrier for devices emitting stable, continuous-wave, single-frequency radiation at $523.5 \mathrm{~nm}$. Large-scale holography and pumping of IR, CW optical-parametric oscillators are just two of the applications that are addressed with this product, says the manufacturer. The company expects to expand its experience in all-solid-state, single-frequency lasers into other spectral regions with the development of daughter products and variants of the GreenLine 1000.

Reader Enquiry No. 106

\section{ILT 6800}

From Ion Laser Technology

An argon ion laser that offers 1,000 MW power

Available in the UK from AG ElectroOptics, the 6800 series provides over $1 \mathrm{~W}$ of power in a multi-line configuration. Singleline or tunable output from $457 \mathrm{~nm}$ to $514 \mathrm{~nm}$ are also available. The transverse beam mode is $\mathrm{TEM}_{00}$, with higher order modes available on multi-line models. Beam noise is less than one per cent from DC to $2 \mathrm{MHz}$, and pointing stability is better than 10 microradians after warm-up. According to the manufacturer, the 6800 offers versatility in applications by virtue of the precision light feedback mechanism, which monitors the laser output and amplitude stability, and through the use of a robust power supply with power-factorcorrection circuitry. The water jacket for tube cooling is said to eliminate minute vibrations caused by acoustic coupling from cooling fans, and extends the useful life of the tube. The resonator design is said to provide a wide range of fine adjustment of beam parameters with improved stability. Optional features include a remote control with standby interlock, a quick-change feature from single-line to multi-line operation and a digital interface for remote diagnostics or host-based control. The 6800 series is stated to meet international regulatory standards including the CE mark.

Reader Enquiry No. 107

Laser accessories

LDC-3700 controlle!

From ILX Lightwave

Precision laser diode controllers representing the next generation in their family of

precision laser diode controllers

New features include a new processor platform that allows a more robust, high-speed GPIB interface for demanding remote-control operations. Each of the LDC-3700 precision current controller sources provides selectable constant-current power operation, an analog modulation capability, as well as triggering functions for complete system solutions. These new instruments have been redesigned for an expanded dual-current range and are available with maximum currents from $100 \mathrm{~mA}$ to 4,000 $\mathrm{mA}$ for driving a variety of laser diodes. The TEC controllers offer $16 \mathrm{~W}$ of control power with a choice of temperature sensors for improved precision temperature control.

Reader Enquiry No. 108

Flipper mount mirror
From New Focus
This motorized mount allows the automation
of multiple experiments

The company has added a motor to their Flipper mirror mount, which now lets users repeatedly flip a mirror in and out of a beam path remotely. A single $9-\mathrm{V}$ battery provides hundreds of flips, and the unit can be controlled through a TTL level input. Manual alignment screws are provided for adjusting both the tip and the tilt of the mirror. These mirrors should prove useful for applications where users need to share laboratory equipment. Also available is the stackable Flip-Up optics mount. The model 9897 FlipUp mount has been designed to allow easy addition and removal of filters and lenses from experiments. The mount can be easily
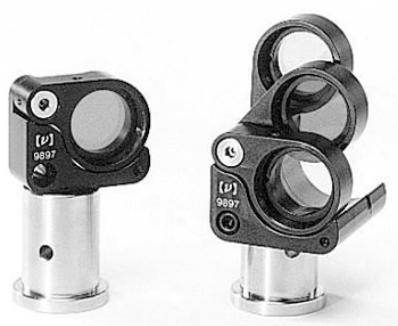

Also available from New Focus are Flip-Up optic mounts for easy changing of lenses and filters.

stacked to change filter values quickly. Both thin and thick optics are held firmly using either a threaded Delrin insert or a standard set screw.

Reader Enquiry No. 109

\section{UVS laser accessory}

From Laser Sciences

An accessory that has been developed to provide economical ultraviolet laser sources

When coupled to the company's pulsed nitrogen laser and laser dye module, this accessory produces wavelength selectable light from $220 \mathrm{~nm}$ to $320 \mathrm{~nm}$. This is a compact unit, which is said to be suitable for ultraviolet laser-induced fluorescence applications in clinical, pharmaceutical, environmental and process control work.

\section{Reader Enquiry No. 110}

\section{Internetresource}

Located on the World Wide Web, Tate Web offers information and links to optics and photonics resources: $\langle w w w$.tateweb.com $\rangle$. $\square$

These notes are compiled by Brendan Horton from information provided by the manufacturers. For more details, fill in the reader service card bound inside the journal.

\section{ADVERTISEMENT}

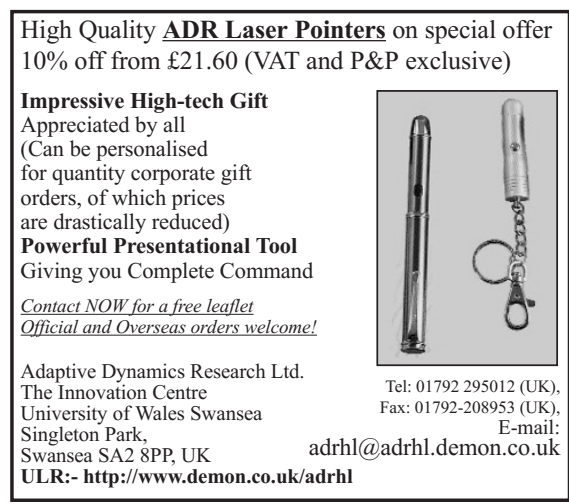

